
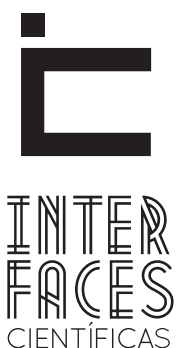

SAÚDE E AMBIENTE

ISSN IMPRESSO 2316-3313

E - ISSN 2316-3798

DOI - 10.17564/2316-3798.2017v5n3p47-56

\title{
INTENSIDADE E SINTOMAS DEPRESSIVOS EM USUÁRIOS DA ESTRATÉGIA SAÚDE DA FAMÍLIA
}

\author{
INTENSITY AND DEPRESSIVE SYMPTOMS IN USERS OF THE FAMILY HEALTH STRATEGY \\ LA INTENSIDAD DE SÍNTOMAS DEPRESIVOS EN LOS USUARIOS DE LA ESTRATÉGIA SAÚDE DA FAMÍLIA
}

Tarciana Sampaio Costa ${ }^{1}$

Milena Nunes Alves de Sousa ${ }^{3}$

Francisco Arnoldo Nunes de Miranda ${ }^{5}$
Raquel Campos de Medeiros ${ }^{2}$

Ricardo Riyoiti Uchida ${ }^{4}$

\section{RESUMO}

A depressão se apresenta como um grave problema de saúde pública, com efeitos físicos e mentais. Assim sendo, objetivou-se averiguar a intensidade e os sintomas depressivos em usuários da Estratégia Saúde da Família, do município de Abaiara, Ceará, Brasil. Estudo de campo, com abordagem quantitativa, realizado com 64 usuários adscritos à Estratégia (67,4\% do total de usuários com sintomas de depressão). Para a coleta de dados foi utilizado o Inventário de Beck, o qual avalia 21 itens, aplicado a partir de visitas domiciliares entre abril e junho de 2010. Os dados foram analisados mediante análise estatística descritiva. Dentre os itens pesquisados, identificou-se que $8(38,1 \%)$ foram expressos com intensidade máxima, $5(23,8 \%)$ com intensidade média, $3(14,3 \%)$ com intensidade baixa e $5(23,8 \%)$ com inten-

sidade neutra. Considerando os sintomas com intensidade máxima, destacaram-se a tristeza $(48,4 \%)$, distúrbio do sono $(48,4 \%)$, retração social $(39,1 \%)$, preocupação somática $(37,5 \%)$, indecisão $(34,4 \%)$, pessimismo $(31,3 \%)$ e sensação de fracasso $(28,1 \%)$. Os resultados demonstram entre os sintomas pesquisados apresentaram intensidades máxima e média para a maioria dos itens analisados. Contudo, destacaram-se tristeza, retração social e distúrbio do sono; autodepreciação, autoacusação e irritabilidade, respectivamente.

\section{PALAVRAS CHAVE}

Saúde Mental, Depressão, Atenção Primária à Saúde. 


\section{ABSTRACT}

Depression appears as a serious public health problem, with physical and mental effects. Therefore, it aimed to determine the intensity and depressive symptoms in users of the Family Health Strategy, the municipality of Abaiara, Ceará, Brazil. Field of study, with a quantitative approach, conducted with 64 users ascribed to the strategy ( $67.4 \%$ of total users with symptoms of depression). For data collection was used the Beck Inventory, which assesses 21 items, applied from home visits between April and June 2010. Data were analyzed using descriptive statistics. Among the items surveyed, it was found that $8(38.1 \%)$ were expressed with maximum intensity, 5 (23.8\%) with medium intensity, 3 (14.3\%) with low intensity and $5(23.8 \%)$ with neutral intensi- ty. Considering the symptoms with maximum intensity, they stood out the sadness (48.4\%), sleep disturbance (48.4\%), social withdrawal (39.1\%), somatic concern (37.5\%), indecision (34 4\%), pessimism (31.3\%) and sense of failure $(28.1 \%)$. The results show between symptoms presented maximum and average intensities for most items analyzed. However, they stood out sadness, social withdrawal and sleep disorders; self-deprecation, self-accusation and irritability, respectively.

\section{KEYWORDS}

Mental Health, Depression, Primary Health Care.

\section{RESUMEN}

La depresión aparece como un problema grave de salud pública, con efectos físicos y mentales. Por lo tanto, el objetivo es determinar la intensidad y los síntomas depresivos en los usuarios de la Estratégia Saúde da Família, del municipio de Abaiara, Ceará, Brasil. Trabajo de campo, con un enfoque cuantitativo, realizado con 64 usuarios adscritos al programa (el $67,4 \%$ del total de usuarios con los síntomas de la depresión). Para la recolección de datos se utilizó el Inventario de Beck, que evalúa 21 elementos, aplicado a partir de las visitas a domicilio, entre abril y junio de 2010. Los datos fueron analizados utilizando estadística descriptiva. Entre los artículos estudiados, se encontró que $8(38,1 \%)$ se expresaron con la máxima intensidad, $5(23,8 \%)$ de intensidad media, $3(14,3 \%)$ con baja intensidad y $5(23,8 \%)$ con intensidad neu- tral. Teniendo en cuenta los síntomas con la máxima intensidad, se destacaban la tristeza $(48,4 \%)$, trastornos del sueño (48,4\%), el aislamiento social (39,1\%), la preocupación somática (37,5\%), la indecisión (34 $4 \%)$, el pesimismo $(31,3 \%)$ y la sensación de fracaso $(28,1 \%)$. Los resultados muestran que los síntomas presentaran intensidades máximas y medias para la mayoría de los elementos analizados. Sin embargo, se destacaban tristeza, trastornos del sueño y la abstinencia social; autodesprecio, la autoacusación y la irritabilidad, respectivamente.

\section{PALABRAS CLAVE}

Salud mental, Depresión, Atención primaria de salud. 


\section{INTRODUÇ̃̃O}

Segundo as diretrizes da Organização Mundial de Saúde (OMS) a área de Saúde Mental constitui uma das principais prioridades no âmbito da Saúde Pública, tanto pelo seu envolvimento direto com os serviços de saúde da assistência primária, quanto pela questão epidemiológica, que vem se agravando com o passar dos anos de maneira considerável na atenção primária à saúde. Estima-se que até 2020 as doenças mentais ocupem o segundo lugar no ranking de doenças incapacitantes (MOLINA et al.,2012).

Entre os agravos que merecem destaque, pode-se citar a depressão, a qual se apresenta nos dias atuais como um grave problema de saúde pública, evidenciada como a mais comum dentre as doenças mentais e, por ser incapacitante, envolve limitações na vida pessoal, profissional, social e econômica (ABELHA, 2014).

É apontada, ainda, como uma das dez principais causas de incapacitação no mundo, com limitações do funcionamento físico, pessoal e social (NORONHA JÚNIOR et al., 2015). No Brasil estima-se que a prevalência da depressão varia entre $9,7 \%$ a $16,1 \%$ na população geral, tendo a mulher um percentual mais elevado, correspondendo a $(21,8 \%)$ do que em homens (8,9\%). Destaca-se que estes resultados foram identificados em um estudo realizado em uma população de usuários de um serviço de atenção primária, onde aponta alta prevalência de sintomas depressivos (CUNHA; BASTOS; DUCA, 2012).

Sua característica insidiosa vai destruindo as esperanças e o brilho da vida de seus portadores [...]. A depressão se caracteriza pela perda de interesse e prazer por tudo, pelo sentimento de tristeza e baixa da autoestima. Os quadros mais graves podem levar ao suicídio. Apesar disso, a doença permanece escondida e não tratada. (ABELHA, 2014, p. 223).

Contudo, seu rastreamento constitui-se como principal medida para garantia de um tratamento adequado ao usuário com vistas a assegurar um maior acesso aos serviços de saúde de cuidados primários, desta maneira, refletindo-se de forma positiva na redução de custos, com a realização de exames desnecessários e minimização dos efeitos decorrentes de quadros depressivos extremos (APÓSTOLO et al., 2011).

Entretanto, segundo Molina e outros autores (2012), cerca de $50 \%$ a $60 \%$ dos casos de depressão acabam sendo negligenciados pela dificuldade em receber tratamento específico, pela presença de comorbidades associadas aos sintomas e dificuldades por parte da equipe de atenção primária em diagnosticá-la.

0 quadro perdura mesmo com a criação do Núcleo de Apoio a Saúde da Família (NASF), o qual tem possibilitado avanços na área da Saúde Mental na Atenção Primária. Tendo como princípios fundamentais: territorialização, intersetorialidade, reabilitação psicossocial, multiprofissionalidade, interdisciplinaridade, desinstitucionalização, promoção da cidadania e construção da autonomia; buscando realizar uma melhor articulação nas melhorias voltadas ao público alvo, como também diminuição da fragmentação de especialidades voltadas à atenção primária, fazendo com que a equipe pense no adoecimento nas suas diversas variantes e, deste modo, desmistificando o modelo tradicional de tratamento dos transtornos mentais centrados ao Hospitalar (CAMPOS et al., 2011).

A frequência elevada da depressão, as consequências negativas sobre a qualidade de vida dos indivíduos, relacionamento pessoal, familiar e social, merecem um cuidado especial pela rede de Atenção Básica. Seguindo uma das recomendações da OMS onde a atenção primária tem fundamental relevância no que diz respeito ao desenvolvimento de ações focadas no rastreamento e monitoramento desse agravo (MADEIRA et al., 2013).

Ao partir do pressuposto de que a saúde mental, especificamente a depressão, deve ser abordada desde a atenção básica e que este âmbito é propício a receber usuários apresentando transtornos, caracterizando uma demanda da Estratégia de Saúde da Família (ESF), faz-se necessária investigar a problemática a partir da seguinte inquietação: quais os sintomas depressivos apresentados entre os usuários da ESF?

Desse modo, considera-se relevante a análise dos aspectos que permeiam a depressão na Atenção Bási- 
ca. Assim, este estudo objetivou averiguar a intensidade e os sintomas depressivos em usuários da ESF, do município de Abaiara, Estado do Ceará (CE).

\section{MÉTODO}

Estudo de campo, do tipo descritivo, com abordagem quantitativa, realizado em quatro Unidades Básicas de Saúde da Família, do município de Abaiara-Ceará, sendo duas na zona rural e duas na zona urbana. A população desta pesquisa consistiu-se dos usuários adscritos à ESF, obedecendo aos seguintes critérios de inclusão: indivíduos com faixa-etária a partir de 20 anos de idade, com sintomas depressivos, em condições de diálogo e domicílio acessível para visitas domiciliares. Excluíram-se os sujeitos que não estavam cadastrados nas Unidades Básicas de Saúde da Família selecionadas para pesquisa.

A coleta dos dados transcorreu nos meses de abril, maio e junho de 2010, a partir do contato prévio com 22 Agentes Comunitários de Saúde (ACS), inseridos nas quatro equipes da ESF, os quais informaram os usuários que atendiam aos critérios outrora estabelecidos neste estudo, bem como o local de residência para posterior coleta de dados. Portanto, a amostra foi do tipo não probabilista intencional, sendo composta por 64 usuários $(67,4 \%$ do total de usuários com sintomas de depressão do município de Abaiara-Ceará). Utilizou-se como instrumento dessa coleta o Inven- tário de Beck - Depression Inventory Scales, validada em Português em uma população de estudantes universitários (GORENSTEIN; ANDRADE, 1998). Compõe-se por 21 quesitos, contendo quatro afirmativas cada um, incluindo sintomas e atitudes com intensidade variável entre 0 e 3 , sendo zero a intensidade neutra e três a intensidade máxima. As pontuações do questionário podem variar entre 0 e 63 em sua totalidade. Salienta-se que este instrumento é de grande valia, pois serve para se observar o traço latente, considerando variáveis secundárias. Porém, tal escala não foi construída para fins diagnósticos, mas para complementar entrevistas clínicas, atendendo a visão do próprio paciente (CASTRO; TRENTINI; RIBOLDI, 2010; GORENSTEIN et al., 2011).

Optou-se pela análise estatística descritiva com a utilização do programa Microsoft Office Excel como subsídio para a construção das tabelas e gráficos. Ressalta-se que a pesquisa foi aprovada pelo Comitê de Ética da Faculdade Santa Maria, por meio do protocolo de $n^{0} 3761209$, o que garantiu o respeito a normativa de pesquisa envolvendo seres humanos.

\section{RESULTADOS}

A Tabela 1 apresenta a frequência da escolha de cada uma das 21 questões do inventário de depressão de Beck, em número absoluto e em porcentagem, de acordo com a intensidade, variando de neutra a máxima.

Tabela 1 - Distribuição da frequência e porcentagem dos itens do inventário de depressão de Beck entre os usuários da ESF. Abaiara-CE, Brasil, 2011

\begin{tabular}{lllll}
\hline Itens & Intensidade neutra & Intensidade baixa & Intensidade média & Intensidade máxima \\
\hline Tristeza & $10(15,6 \%)$ & $12(18,8 \%)$ & $11(17,2 \%)$ & $31(48,4 \%)$ \\
Pessimismo & $17(26,6 \%)$ & $12(18,7 \%)$ & $15(23,4 \%)$ & $20(31,3 \%)$ \\
Sensação de fracasso & $13(20,3 \%)$ & $16(25,0 \%)$ & $17(26,6 \%)$ & $18(28,1 \%)$ \\
Falta de satisfação & $12(18,8 \%)$ & $21(32,8 \%)$ & $18(28,1 \%)$ & $13(20,3 \%)$ \\
Sensação de culpa & $31(48,4 \%)$ & $11(17,2 \%)$ & $12(18,8 \%)$ & $10(15,6 \%)$ \\
Sensação de punição & $30(46,9 \%)$ & $05(7,8 \%)$ & $06(9,4 \%)$ & $23(35,9 \%)$ \\
\hline
\end{tabular}




\begin{tabular}{lllll}
\hline Itens & Intensidade neutra & Intensidade baixa & Intensidade média & Intensidade máxima \\
\hline Autodepreciação & $19(29,7 \%)$ & $12(18,8 \%)$ & $26(40,6 \%)$ & $07(10,9)$ \\
Autoacusação & $13(20,3 \%)$ & $17(26,6 \%)$ & $28(43,7 \%)$ & $06(9,4 \%)$ \\
Ideias suicidas & $38(59,4 \%)$ & $12(18,7 \%)$ & $08(12,5 \%)$ & $06(9,4 \%)$ \\
Crises de choro & $11(17,2 \%)$ & $12(18,7 \%)$ & $25(39,1 \%)$ & $16(25 \%)$ \\
Irritabilidade & $09(14,1 \%)$ & $11(17,2 \%)$ & $28(43,7 \%)$ & $16(25 \%)$ \\
Retração Social & $17(26,6 \%)$ & $15(23,4 \%)$ & $07(10,9 \%)$ & $25(39,1)$ \\
Indecisão & $19(29,7 \%)$ & $06(9,4 \%)$ & $17(26,5 \%)$ & $22(34,4 \%)$ \\
Aparência física & $24(37,5 \%)$ & $11(17,2 \%)$ & $14(21,9 \%)$ & $15(23,4 \%)$ \\
Inibição para o trabalho & $19(29,7 \%)$ & $21(32,8 \%)$ & $17(26,6 \%)$ & $07(10,9 \%)$ \\
Distúrbio do sono & $08(12,5 \%)$ & $11(17,2 \%)$ & $14(21,9 \%)$ & $31(48,4 \%)$ \\
Fadiga & $14(21,9 \%)$ & $19(29,7 \%)$ & $19(29,7 \%)$ & $12(18,7 \%)$ \\
Perda do apetite & $18(28,1 \%)$ & $20(31,3 \%)$ & $13(20,3 \%)$ & $13(20,3 \%)$ \\
Perda de peso & $21(32,8 \%)$ & $15(23,4 \%)$ & $10(15,6 \%)$ & $18(28,2 \%)$ \\
Preocupação somática & $06(9,4 \%)$ & $20(31,2 \%)$ & $14(21,9 \%)$ & $24(37,5 \%)$ \\
Diminuição da libido & $17(36,6 \%)$ & $13(20,3 \%)$ & $13(20,3 \%)$ & $21(32,8 \%)$ \\
\hline
\end{tabular}

Fonte: Dados da pesquisa.

Constatou-se que os itens "tristeza", "pessimismo", "sensação de fracasso", "retração social", "indecisão", "distúrbio do sono" e "preocupação somática", obtiveram porcentagens superiores na intensidade máxima, o que indica a maior frequência destes sintomas e atitudes na amostra estudada.

Em seguida, observaram-se os sintomas que apresentaram valores referentes à intensidade média, entre os quais a "autodepreciação", "autoacusação", "crises de choro", "irritabilidade" e "fadiga". A ausência de "satisfação", "inibição para o trabalho" e "perda de apetite" obtiveram porcentagens de intensidade baixa e a "sensação de culpa", "sensação de punição", "ideias suicidas", "aparência física" e "perda de peso", porcentagens de intensidade neutra.

Ademais, os achados indicaram as seguintes intensidades: neutra $(23,8 \%)$ o que corresponde a inexistência de sintomas depressivos, caracterizando a não ocorrência da doença; baixa (14,3\%), o que corresponde as primeiras manifestações da Depressão, ainda na sua fase inicial, sendo este estado indicativo de intervenções em menor complexidade no intuito de evitar o agravamento do caso, para tanto, utiliza-se recursos mais simples, a exemplo da Psicoterapia e atividade física, sem necessariamente a intervenção farmacológica e apresenta ótimo prognóstico; média $(23,8 \%)$, o que corresponde ao agravamento da doença, tendo em vista a ausência de intervenções no início da apresentação dos sintomas.

E alta (38,15\%), o que caracteriza a negligência com os cuidados primários e secundários da Depressão, fase esta a qual apresenta as piores manifestações clínicas, as quais correspondem a quase todos os sintomas descritos na Tabela 1 e requer as intervenções de alto nível de complexidade, a exemplo da internação.

\section{DISCUSSÃO}

Os sintomas de intensidade máxima identificados neste estudo, quais sejam tristeza, pessimismo, sensação de fracasso, retração social, indecisão, distúrbio do sono e preocupação somática, totalizaram $38 \%$ dos itens do inventário de Beck, sendo estes 
apresentados pelos usuários da ESF do município de Abaiara-CE e vivenciados mais intensamente. Esses sentimentos são característicos da depressão e quando apresentados pela população adscrita, a equipe deve ser conhecedora destes aspectos que permeiam o sofrimento do paciente depressivo, sendo capaz de identificá-los, precocemente, no intuito de traçar um plano de cuidados ou encaminhar ao atendimento especializado, quando necessário.

Vale destacar que, os sintomas da depressão são conhecidos desde a antiguidade (500 a.C. - 100 d.C.), quando esta ainda era conhecida como melancolia, dentre estes, citam-se a tristeza, perda de sono, falta de apetite, perda da capacidade de amar e desejo de morte. Somente a partir da metade do século XX, quando em estudos mais aprofundados no campo descritivo das doenças mentais houve largos avanços por meio das características biológicas e da ação de substâncias químicas, identificando-se que a melancolia nada mais era que um subtipo da depressão (DUAILIB et al., 2015).

Atualmente, são muitos sintomas identificados como humor depressivo, baixa autoestima, perda de interesse, fadiga, alterações na concentração e falta de apetite, todos permeados basicamente pela tristeza, a qual o paciente se depara com um descontrole de sentimentos, em que a felicidade, apesar de desejada, torna-se inalcançável (RIOS; BARBOSA; BELASCO, 2010; ABELHA, 2014).

Quanto à visão pessimista diante do futuro e o sentimento duradouro de fracasso, as comorbidades associadas ao quadro, relacionam-se com a avaliação negativa de si mesmo, do mundo e do futuro, desta maneira a tristeza pode aparecer como um sinal de alerta, onde o acometido necessita de auxílio expresso, uma vez que as novas perspectivas futuras se anulam, fazendo com que ultrapasse o limiar da normalidade (CAVALCANTE, MINAYO; MANGAS, 2013; SANTOS et al., 2010).

Diante desses sentimentos, os relacionamentos interpessoais são comprometidos, havendo retração social e alteração na sua vivência uma vez que a pessoa depressiva não se interessa pelos seus passatempos prediletos, com episódios de insegurança e frustração o seu convívio social acaba se tornando cada vez mais desinteressado (RIBEIRO; NASCIMENTO; COUTINHO, 2010; ABELHA, 2014). Neste aspecto, o enfermeiro da ESF pode intervir na promoção da verbalização, avaliação dos fatores contribuintes dos sentimentos negativos e na escuta atenta das preocupações negativas (SANTOS et al., 2010).

Na pessoa deprimida, há uma diminuição da capacidade de pensar, de se concentrar ou de tomar decisões, sendo este sentimento expressado com grande intensidade pelos usuários da ESF do município de Abaiara-CE. Decisões antes quase automáticas parecem agora custar esforços intransponíveis. Outro ponto a se observar é que os episódios são marcados de maneira aleatória, havendo uma tendência a recidivas; podendo ocorrer, ainda, um déficit cognitivo, geralmente, em função da fadiga e dificuldade de atenção, além do desinteresse generalizado.

Alterações na qualidade do sono têm sido relatadas em diversos estudos científicos, isso se dá especificamente pelo fato de todo o envolvimento biopsiquicosocial que a depressão traz ao indivíduo. Tendo como sintoma principal e que deve ser investigada pelos profissionais da ESF, a insônia, que se apresenta mais tipicamente, intermediária (acordar no meio da noite, com dificuldades para voltar a conciliar o sono), ou terminal (acordar mais precocemente pela manhã) (FERNANDES; NASCIMENTO; COSTA, 2010).

A depressão pode ser avaliada em diversas dimensões, mas o que se tem que observar é a larga proporção e grande importância que esse termo tem ganhado na sociedade como um todo e em nosso cotidiano dia após dia (MARTIN et al., 2012).

Os sintomas de intensidade média identificados neste estudo, qual seja a autodepreciação, autoacusação, crises de choro, irritabilidade e fadiga, totalizaram $24 \%$ dos itens do inventário de Beck. A autodepreciação está relacionada à presença de sentimento de desapontamento ou desgosto de si próprio, ou seja, a insatisfação com o eu. Este sentimento deve ser trabalhado pela equipe da ESF, no intuito de motivar a autoestima ao paciente (CAVALCANTE; MINAYO; MANGAS, 2013). 
No que diz respeito à autoacusação, a base da grande tortura do autojulgamento é a frustração pela distância entre o que se não é e a imagem de como deveria ser. Segundo Cavalcante, Minayo e Mangas (2013) a autocrítica associada ao sentimento de culpabilidade pode ser entendida como uma barreira que afeta o prazer e o livre fluir de determinadas situações.

Os sentimentos negativos que perpetuam na vida do depressivo, provocam crises de choro, e quando em estágios mais avançados da doença, o depressivo não consegue chorar, ainda que deseje, devendo este receber tratamento especializado. Quando o deprimido consegue expressar suas angústias e tristezas, 0 choro é o modo que este se utiliza para demonstrar e descarregar tais sentimentos (GOMES, 2011). Tendo a família um importante papel na compreensão dos sintomas o qual o indivíduo se encontra (NOGUEIRA et al., 2011; OLIVEIRA et al., 2011; SILVA et al., 2016). Assim, a ESF deve estabelecer vínculo e desenvolver ações que abranjam também a família do usuário.

Ainda de acordo com Gomes (2011) a depressão pode provocar fadiga persistente ou sensação de perda de energia, mesmo sem esforço físico, e com ela as atividades mais leves parecem exigir esforço substancial. Para Santos e outros autores (2010) o enfermeiro, atuando na ESF, pode intervir na tentativa de minimizar este sintoma, transmitindo empatia, ajudando este cliente a identificar pontos importantes e vantagens pessoais, disponibilizando tempo para que o mesmo expresse sentimentos, encorajando-o a perguntar e auxiliar na identificação do que ele pode fazer por si.

Os sintomas de intensidade baixa identificados neste estudo, qual seja a falta de satisfação, inibição para o trabalho e perda do apetite, totalizaram 14\% dos itens do inventário de Beck. 0 deprimido apresenta alterações do apetite. Muitas vezes, a pessoa precisa esforçar-se para comer ou ser ajudada por terceiros a se alimentar. Algumas formas específicas de depressão são acompanhadas de aumento do apetite, que se mostra, caracteristicamente, aguçado por carboidratos e doces (GOMES, 2011).

Os sintomas de intensidade neutra identificados neste estudo, qual seja a sensação de culpa, sensação de punição, ideias suicidas, aparência física e perda de peso, totalizaram $23,8 \%$ dos itens do inventário de Beck, assinalados pelos usuários da ESF do município de Abaiara-CE.

Apesar da ideia suicida se apresentar de intensidade neutra, este resultado pareceu preocupante, uma vez que ideias suicidas se fazem presente em depressão de nível elevado e são expressas nas demais intensidades, baixa, média e máxima, perfazendo um total de $26(40,6 \%)$ dos usuários entrevistados.

Os pensamentos de morte podem ser detectáveis a partir do pessimismo e do olhar negativo em relação à vida e ao futuro, bem como do surgimento de preocupações sérias, por parte do paciente, a respeito de pessoas próximas e queridas, refletidas como medo da separação e acompanhadas de grande ansiedade. Destaca-se que o medo de o paciente tentar o suicídio, enfatiza os aspectos de vigilância, mas não os da atenção psicológica. 0 estado deprimido e a presença de comorbidades psiquiátricas associadas funcionam como um trampolim para o comportamento suicida, onde a autoimpulsividade pressiona tal comportamento. Por isso, os pensamentos relativos à morte devem ser sistematicamente investigados, uma vez que essa conduta poderá prevenir atos suicidas, dando ensejo ao doente de se expressar a respeito (CAVALCANTE; MINAYO; MANGAS, 2013).

Em relação à aparência física, a insatisfação da própria imagem é decorrente da baixa autoestima, fazendo com que o deprimido não se sinta atraente ou considere-se feio. Ao considerar que a natureza da depressão possui uma dimensão multifatorial, tendo origem influenciada tanto por fatores endógenos como exógenos, todos esses sintomas psíquicos, fisiológicos e comportamentais se apresentam de diversas maneiras, em termos de grau e intensidade. Cada pessoa possui sua maneira de ser e de se apresentar diante de um quadro depressivo (FERNANDES; NASCIMENTO; COSTA, 2010).

Para tanto, o que se observa na prática é por meio da atenção primária é o insuficiente preparo de profissionais para lidar com estes sintomas e atitudes apresentados pelos portadores de depressão. Em um 
estudo clínico pesquisas apontam que o diagnóstico da depressão pode ser negligenciado pela presença de sinais e sintomas que podem ser facilmente confundidos com doenças preexistentes, desta forma é necessário que a equipe como um todo tenha uma abordagem inclusiva, observando cada sintoma independentemente de uma patologia já existente (VENTURA, 2011).

Ao ponderar que os cuidados com os doentes mentais que necessitam de atenção diária não se resumem à medicação, a problemática da depressão na atenção primária não se limita apenas ao diagnóstico. A intervenção abrange busca ativa, detecção precoce, acompanhamento de suporte social e promoção de oficinas terapêuticas, o que exige do enfermeiro estratégias de rastreamento dos problemas mentais, aconselhamento para indivíduos e famílias, grupos de autoajuda, visitas domiciliares e demais intervenções intensivas em situações de alto risco. Contudo, ainda se encontra bastante necessidade no que diz respeito à inclusão de medidas políticas por parte do governo direcionadas ao campo da Saúde Mental, necessitando da participação das equipes e sobretudo da população em prol de melhorias na qualidade da prestação de serviços voltados à prevenção, promoção e recuperação da saúde do indivíduo mentalmente adoecido (SOBRAL; CAMPOS, 2012).

\section{CONCLUSÃO}

Os resultados demonstram entre os sintomas pesquisados intensidades máxima e média para a maioria dos itens analisados. Contudo, destacaram-se tristeza, retração social e distúrbio do sono; autodepreciação, autoacusação e irritabilidade, respectivamente.

Os achados sugerem a necessidade urgente de intervenções apropriada no campo da saúde mental na Atenção Básica. Diante de tais lacunas, urge a necessidade de investimentos e incentivos específicos por parte da União e do Estado no referente ao Piso da Atenção Básica (PAB), fomentando a abordagem da saúde mental aliada à ESF, na perspectiva de reversão do atual quadro do município de Abaiara-CE.

\section{REFERENCIAS}

ABELHA, L. Depressão, uma questão de saúde pública. Cad. Saúde Colet., Rio de Janeiro, v.22, n.3, p.223, 2014.

APÓSTOLO, J.L.A. et al. Depressão, ansiedade e estresse em usuários de cuidados primários de saúde. Rev. Latino-Am. Enfermagem, Ribeirão Preto, v.19, n.2, p.1-6, 2011.

CAMPOS, R.O. et al. Saúde mental na atenção primária à saúde: estudo avaliativo em uma grande cidade brasileira. Ciên. Saúde Colet., Rio de Janeiro, v.16, n.12, p.4643-4652, 2011.

CUNHA, R.V.; BASTOS, G.A.N.; DUCA, G.F.D. Prevalência de depressão e fatores associados em comunidade de baixa renda de Porto Alegre, Rio Grande do Sul. Rev. Bras. Epidemiol., Rio de Janeiro, v.15, n.2, p.346-354, 2012.

CAVALCANTE, F.G.; MINAYO, M.C.S.; MANGAS, R.M.N. Diferentes faces da depressão no suicídio em idosos. Ciên. Saúde Colet., Rio de Janeiro, v.18, n.10, p.2985-2994, 2013.

FERNANDES, M.G.M.; NASCIMENTO, N.F.S.; COSTA, K.N.F.M. Prevalência e determinantes de sintomas depressivos em idosos atendidos na atenção primária de saúde. Rev Rene, Fortaleza, v.11, n.1, p.19-27, 2010

GOMES, A.M.A. Um olhar sobre depressão e religião numa perspectiva compreensiva. Estudos de Religião, São Bernardo do Campo, v.25, n.40, p.81-109, 2011.

GORENSTEIN, C.; ANDRADE, L. Inventário de depressão de Beck: propriedades psicométricas da versão em português. Rev Psiq. Clín., São Paulo, v.25, n.5, p.23-31, 1998. 
GORENSTEIN, C. et al. Manual inventário de depressão de Beck-BDI-II. São Paulo: Casa do Psicólogo, 2011.

MADEIRA T.C.S. et al. Depressão em idosos hipertensos e diabéticos no contexto da Atenção Primária em Saúde. Rev. APS., Juiz de Fora, v.16, n.4, p.393398, 2013.

MARTIN, D. et al. Significado da busca de tratamento por mulheres com transtorno depressivo atendidas em serviço de saúde público. Interface (Botucatu), Botucatu, v.16, n.43, p.885-899, 2012.

MOLINA, M.R.A.L. et al. Prevalência de depressão em usuários de unidades de atenção primaria. Rev. Psiq. Clín., São Paulo, v.39, n.6, p.194-197, 2012.

NOGUEIRA, J.A. et al. Enfoque familiar e orientação para a comunidade no controle da tuberculose. Rev. Bras. Epidemiol., Rio de Janeiro, v.14, n.2, p.207216, 2011.

NORONHA JÚNIOR, M.A.G. et al. Depressão em estudantes de medicina. Rev. Med. Minas Gerais, Belo Horizonte, v.25, n.4, p.562-567, 2015.

OLIVEIRA, M.G. et al. O doente em tratamento de tuberculose no município de Itaboraí, Rio de Janeiro - participação da família. Rev. Bras. Med. Fam. Comum., Rio de Janeiro, v.6, n.18, p.40-45, 2011.
RIBEIRO, K.C.S.; NASCIMENTO, E.S.; COUTINHO, M.P.L. Representação Social da Depressão em uma Instituição de Ensino da Rede Pública. Psicol. Ciênc. Prof., Brasília, v.30, n.3, p.448-63, 2010.

RIOS, K.A.; BARBOSA, D.A.; BELASCO, A.G.S. Avaliação de qualidade de vida e depressão de técnicos e auxiliares de enfermagem. Rev. Latino-Am. Enfermagem, Ribeirão Preto, v.18, n.3, p.1-9, 2010.

SANTOS, S.S.C. et al. Diagnósticos e intervenciones de enfermería para ancianos con depresión y residentes en una institución de larga estancia (ILE). Enferm. Global, Murcia, n.20, p.1-14, 2010.

DUAILIB, K.; SILVA, A.S.M.; JUBARA, C.F.B. Depressão. Rev. Bras. Med., São Paulo, v.72, n.12, p.40-41, 2015.

SILVA, C.S. et al. Frequência de casos positivos para tuberculose em Tobias Barreto-SE no período de 2010-2015. Interfaces Cient. Saúde Amb., Aracaju, v.4, n.2, p.29-34, 2016.

SOBRAL, F.R.; CAMPOS, C.J.G. O enfermeiro e a educação em saúde mental na atenção primária: revisão integrativa. Rev. Eletr. Saúde Mental Álcool Drog. (Ed. port.), São Paulo, v.8, n.2, p.100-107, 2012.

VENTURA, T. Depressão e comorbidade: um caso clínico. Rev Port Clin Geral, Lisboa, v.27, p.36-42, 2011. 
1 Enfermeira; Doutora em Ciências da Saúde pela Santa Casa de São Paulo; Professora do curso de enfermagem nas Faculdades Integradas de Patos, Patos-PB, Brasil. E-mail: tarcianasampaio@yahoo.com.br

2 Enfermeira; Professora nas Faculdades Integradas de Patos; Doutora em Ciências da Saúde pela Santa Casa de São Paulo; Professora do curso de enfermagem nas Faculdades Integradas de Patos, Patos-PB, Brasil. E-mail: raquelfip@hotmail.com

3 Enfermeira; Doutora em Promoção da Saúde pela Universidade de Franca; Docente do Curso de Medicina das Faculdades Integradas de Patos, Patos-PB, Brasil. E-mail: minualsa@hotmail.com

4 Psiquiatra; Doutor em Saúde Mental pela Faculdade de Medicina de Ribeirão Preto, USP. E-mail: ricardouchida@hotmail.com

5 Doutor em Enfermagem pela Universidade de São Paulo, Brasil; Líder do Grupo de Pesquisa da Universidade Federal do Rio Grande do Norte, Brasil. Aceito em: 28 de Outubro de 2016 